\title{
ĐO LƯờNG GIÁ TRI CẢM NHÂN CỦA NGƯờI TIÊU DÙNG ĐỐI VỚI NHÓM SẢN PHẨM TIÊUU DÙNG HÀNG VIỆT NAM CHẤT LƯợNG CAO
}

\author{
LÊ BẢO HÂN, LÊ NAM HẢI \\ Trừ̀ng Đại học Công nghiệp thành phố Hồ Chí Minh; \\ lebaohan@iuh.edu.vn,lenamhai@iuh.edu.vn
}

Tóm tắt. Nghiên cứu này được thực hiện với mục tiêu xác định các yếu tố cấu thành giá trị cảm nhận $(\mathrm{GTCN})$ của người tiêu dùng (NTD) đối với nhóm sản phẩm tiêu dùng hàng Việt Nam chất lượng cao (HVNCLC) từ đó đo lường mức GTCN của NTD đối với nhóm sản phẩm này. Phương pháp nghiên cứu hỗn hợp được nhóm tác giả vận dụng kết hợp cùng với việc vận dụng mô hình cấu trúc tuyến (SEM) để xem xét mối quan hệ giữa chúng. Kết quả nghiên cứu cho thấy mô hình GTCN của NTD đối với nhóm sản phẩm tiêu dùng HVNCLC gồm 3 nhân tố: giá trị cảm xúc, giá trị xã hội và chất lượng cảm nhận, các nhân tố này đều có tác động cùng chiều lên GTCN tổng thể của NTD. Riêng nhân tố giá trị mang tính tiền tệ trong trường hợp này chưa có đủ bằng chứng để kết luận thực sự sự có tác động một cách có ý nghĩa lên giá trị cảm nhận.

Từ khóa. GTCN, chất lượng cảm nhận, giá cả mang tính tiền tệ, giá trị cảm xúc, giá trị xã hội, sản phẩm tiêu dùng, HVNCLC.

\section{MEASURING CUSTOMER' PERCEIVED VALUE TO VIETNAMESE HIGH- QUALITY CONSUMER GOODS}

\begin{abstract}
This research aims to identify the factors that constitute consumers' perceived value to Vietnamese high-quality consumer goods. The study also examines how consumers' perceived value of high quality Vietnamese products were. The Structural equation modeling (SEM) was used for determining whether a certain model is valid and imputing relationships between variables in the research framework. The results show that emotional value, social value and perceived quality have a significant impact on perceived value. Current price was rejected the proposed model as a non-nested alternative to the factor model except for the case.

Key words. perceived value, perceived quality, current price, emotional value, social value, consumer goods, high quality Vietnamese products.
\end{abstract}

\section{GIỚI THIỆU}

Từ những năm cuối thế kỷ 20 khái niệm "GTCN" đã được các nhà nghiên cứu trên thế giới quan tâm đến, nó nổi lên như một yếu tố đóng vai trò quan trọng đối với sự sống còn của tổ chức, doanh nghiệp. GTCN đã thu hút đáng kể sự chú ý của các học giả tiếp thị (Parasuraman and Grewal [14]; Sweeney và Soutar [17]) trong việc hoạch định chiến lược kinh doanh và marketing dựa trên quan điểm khách hàng.

Cũng trên quan điểm này Hiệp Hội Doanh nghiệp HVNCLC ra đời nhằm nâng cao lợi thế cho các sản phẩm Việt Nam chú trọng đến sự đánh giá bình chọn của NTD làm nền tảng xây dựng chiến lược. Trải qua hơn 20 năm từ 1996 chương trình HVNCLC đã cấp chứng nhận cho 474 doanh nghiệp, các doanh nghiệp này đã có được những lợi thế cạnh tranh nhất định so với các sản phẩm khác trên thị trường. Tuy nhiên nhiều chuyên gia marketing nhận định khẩu hiệu "Người Việt Nam ưu tiên dùng hàng Việt Nam" đã lấn át sự định hướng trong việc lựa chọn hàng hóa của khách hàng, Trong khi đó "Chất lượng sản phẩm, giá cả và chế độ hậu mãi mới là những yếu tố quyết định niềm tin và sự lựa chọn của NTD.

Theo thống kê của Bộ Công Thuương, một số ngành hàng chủ chốt có số lượng lớn các thương hiệu được chứng nhận HVNCLC nhưng vẫn chưa khẳng định được chỗ đứng của mình trên thị trường như: ngành sữa thì $75 \%$ thị phần sữa bột trên thị trường nội địa là của các hãng nước ngoài; thị phần quần áo thời trang thì $60-70 \%$ rơi vào tay các doanh nghiệp nước ngoài; thị phần giày dép Việt Nam lép vế trên sân nhà chỉ chiếm $50 \%$. Thực trạng này đã phản ánh những tồn tại rất cần được quan tâm để HVNCLC có 
thể trở thành một thương hiệu quốc gia uy tín, đem đến những giá trị cao cho khách hàng, góp phần thúc đẩy nền kinh tế nước ta phát triển bền vững. Muốn đạt được mục tiêu đó các doanh nghiệp phải gia tăng sức cạnh tranh lâu dài dựa trên GTCN dành cho khách hàng, đây cũng là tôn chỉ cho mọi hoạch định chiến lược của tất cả các doanh nghiệp. Hơn thế nữa, giá trị đích thực của thương hiệu là xuất phát từ NTD, nếu họ có những cảm nhận tốt thì thương hiệu mới có giá trị cao. Một số thương hiệu có được những tình cảm rất tốt của NTD nhưng họ lại không chọn dùng. Ngược lại, một số thương hiệu được chọn dùng thì NTD lại không có những cảm tình tốt. Trong hai trường hợp trên thì thương hiệu đều không mang lại nhiều giá trị cho tổ chức. Chương trình HVNCLC đã có rất nhiều hoạt động để kêu gọi tình cảm của NTD nhưng vẫn chưa khẳng định được chỗ đứng của các sản phẩm quốc gia trên chính thị trường nội địa như đã trình bày ở trên. Vậy làm sao để có thể khắc phục được tình trạng đó? Làm sao để những thương hiệu hàng tiêu dùng HVNCLC tìm được lợi thế so với các mặt hàng nhập khẩu có chất lượng, giá cả cạnh tranh?

Những câu hỏi trên là nội dung quan trọng mà đề tài "Đo lường GTCN của NTD đối với sản phẩm tiêu dùng hàng Việt Nam chất lương cao" tập trung giải quyết để tìm ra những giải pháp giúp các doanh nghiệp có được những quyết định kinh doanh hiệu quả trong thị trường cạnh tranh gay gắt ngày nay.

\section{CƠ SỞ LÝ THUYẾT, MÔ HÌNH VÀ GIẢ THUYẾT NGHIÊN CÚU}

\subsection{Co' sở lý thuyết}

Khái niệm hàng Việt Nam chất lượng cao (HVNCLC) được biết đến khi có sự xuất hiện của Chương trình hàng Việt Nam chất lượng cao. Theo đó, hàng Việt Nam chất lượng cao có thể hiểu là những hàng hóa được Doanh nghiệp Việt Nam sản xuất tại Việt Nam được người tiêu dùng bình chọn và phải đảm bảo minh bạch các tiêu chí: (1) chất lượng hàng hóa; (2) minh bạch trong cạnh tranh bao gồm: nhãn hiệu hàng hóa, xuất xứ hàng hóa; (3) trách nhiệm xã hội bao gồm: vấn đề môi trường, thực hiện nghĩa vụ thuế, đảm bảo quyền lợi cho người lao động, chăm lo cho cộng đồng. Điều này đồng nghĩa với việc chính người tiêu dùng sẽ là người giữ vai trò quan trọng trong việc bầu chọn hàng Việt Nam chất lượng cao. Vì vậy, các doanh nghiêp mong muốn gia tăng sức cạnh tranh lâu dài thì cần phải nỗ lực gia tăng giá trị cảm nhận của người tiêu dùng đối với sản phẩm.

Mặc dù có nhiều thuật ngữ và định nghĩa về GTCN của khách hàng (Zeithaml [13]; Woodruff [19]; Monroe [7]; Woodall [18]); nhưng giữa những định nghĩa của các nhà nghiên cứu này đều có những điểm thống nhất đó là: GTCN của khách hàng thì vốn có sẵn hoặc liên quan đến việc sử dụng sản phẩm / dịch vụ; là những gì được cảm nhận chủ quan của NTD hơn là đánh giá khách quan; những sự nhận thức giá trị đặc trưng liên quan đến sự đánh đổi giữa những gì NTD nhận được với những gì mà họ bỏ ra để có được và sử dụng sản phẩm/dịch vụ đó.

Hiện nay, quan điểm marketing thì xem việc tập trung vào nhu cầu của khách hàng là hiển nhiên. Xu hướng này được áp dụng trong những doanh nghiệp hiện đại thông qua mối quan hệ giữa việc duy trì lợi nhuận trong dài hạn và khách hàng Kotler et $\mathrm{al}$ [15]. Đồng thời, theo [15] đã kết luận rằng ứng dụng của marketing hiện đại đã tạo ra hiệu quả trong GTCN của khách hàng cao hơn. Điều này đã nói lên GTCN của khách hàng là tâm điểm của marketing hiện đại.

Woodall giải thích rằng NTD có thể xác định giá trị trước khi mua, trong khi mua, sau khi mua và sau khi sử dụng. Tính chất và các yếu tố quyết định giá trị dành cho khách hàng có thể thay đổi trong suốt các giai đoạn của quá trình mua tiêu dùng. Trên cơ sở đó Grewal et al [2] phân giá trị dành cho khách hàng được cảm nhận thành các thành phần: (1) giá trị nhận được (acquisition value), (2) giá trị giao dịch (transaction value), (3) giá trị sử dụng (in-use value), và (4) giá trị còn lại (redemption value).

Một số tác giả cho rằng việc nhìn nhận giá trị dành cho khách hàng được cảm nhận chỉ bằng sự đánh đổi giữa chất lượng và giá là quá đơn giản Bolton \& Drew [1], đặc biệt khi sản phẩm không phải là điểm trọng tâm của sự quan tâm mà NTD còn đánh giá nhiều hơn như dịch vụ gia tăng được chuyển giao trong tương quan với giá và sự tương tác trong toàn bộ quá trình của việc đưa ra quyết định mua.

Sheth et al [16] phân biệt 5 thành phần của GTCN: (1) giá trị chức năng (functional value) (những lợi ích thực tế liên quan đến các đặc tính sản phẩm), (2) giá trị xã hội (social value) (những lợi ích xã hội, hình tuợng), (3) giá trị cảm xúc (emotional value) (những lợi ích trải nghiệm, cảm xúc), (4) giá trị tri thức (epistemic value) (những lợi ích thúc đẩy sự ham học hỏi), và (5) giá trị theo tình huống (conditional value) (những lợi ích trong những tình huống đặc biệt). 
Theo nghiên cứu của Lapierre [5] xác định các thành phần của GTCN là các lợi ích có được từ sản phẩm (những giải pháp thay thế, chất lượng sản phẩm, sản phẩm theo yêu cầu), từ dịch vụ (khả năng đáp ứng, tính linh hoạt, tính đáng tin cậy, năng lực kỹ thuật), từ mối quan hệ (hình ảnh, sự tin cậy, sự gắn kết).

Trong khi đó, Seth et al [16] thì lại đưa ra một lý thuyết để giải thích tại sao NTD có quyền lựa chọn những thứ họ làm. Ba ứng dụng đặc trưng của lý thuyết được chứng minh liên quan đến những sự lựa chọn của NTD trong ngành thuốc lá. Lý thuyết được giải thích bao gồm tại sao NTD lựa chọn mua hay không mua (sử dụng hay không sử dụng) thuốc lá, tại sao NTD chọn một loại thuốc lá khác. Kết quả của lý thuyết này nhằm đánh giá hành vi NTD. Lý thuyết này xác định năm nhân tố của giá trị ảnh hưởng đến hành vi lựa chọn của NTD bao gồm: (1) giá trị chức năng (functional vale); (2) giá trị xã hội (social value); (3) giá trị cảm xúc (emotional value); (4) giá trị tri thức (epistemic value); (5) giá trị điều kiện (conditional value).

Dựa vào định nghĩa của Seth et al [16], Sweeney \& Soutar [17] đã phát triển thang đo PERVAL để đo lường GTCN của khách hàng. Theo Sweeney \& Soutar [17], GTCN khách hàng là "một phần của một quá trình không ngừng trong việc duy trì mối quan hệ giữa nhà sản xuất và cửa hàng bán lẻ với một khách hàng mục tiêu". Họ đã bỏ qua giá trị điều kiện và giá trị tri thức trong thang đo của Seth et al [16]. Dựa vào Zeithaml [13], họ đã tách giá trị chức năng thành chất lượng và giá cả và thuyết phục rằng hai thành phần giá trị chất lượng và giá trị theo giá cả tạo ra tác động đến GTCN khác nhau cho những NTD khác nhau. Do đó, thang đo GTCN của khách hàng bao gồm 19 biến quan sát, bốn nhân tố: (1) giá trị chất lượng (thuộc tính); (2) giá trị theo giá cả; (3) giá trị cảm xúc; (4) giá trị xã hội. Thang đo này đã được kiểm ra dựa trên sự nhận thức của NTD về hàng hóa lâu bền và đã được tìm thấy độ tin cậy và sự vững chắc trong tình huống trước khi mua và sau khi mua.

Ở Việt Nam tác giả tìm thấy những đề tài nghiên cứu về GTCN như: "Giá trị dành cho khách hàng được cảm nhận và chiến lược cạnh tranh của Công ty TNHH Gas Petrolimex Sài Gòn”- Nguyễn Ngọc Châu [11]: kết quả cho thấy GTCN được đo lường bởi 7 nhân tố: trong đó các nhân tố giá trị sản phẩm, giá trị dịch vụ, giá trị nhân sự, giá trị hình ảnh có tác động thuận chiều; các nhân tố chi phí bằng tiền, chi phí thời gian, chi phí tinh thần có tác động nghịch chiều; "Nghiên cứu GTCN của khách hàng đối với chuỗi siêu thị Co.op Mart" Nguyễn Thị Hồng Thắm [12]: đề tài chỉ ra 5 nhân tố đo lường và tác động cùng chiều lên GTCN của khách hàng đó là phản ứng cảm xúc, chất lượng cảm nhận, danh tiếng, giá cả tiền tệ và giá cả hành vi; bài báo "Đo luờng GTCN của khách hàng cá nhân đối với sản phẩm xe máy điện tại vùng đông nam bộ" của Lê Văn Hoàng \& Nguyễn Thị Ngọc Huỳnh [6]: bài báo chỉ ra 5 nhân tố tác động cùng chiều và cấu thành GTCN đó là giá trị cảm xúc xã hội, Giá trị giá cả, Giá trị chất lượng, giá trị nhân sự, giá trị lắp đặt. Tuy nhiên chưa có nghiên cứu nào về GTCN đối với sản phẩm tiêu dùng. Tính mới của bài viết thể hiện ở phạm vi nghiên cứu mang tính thời sự trong tình hình cạnh tranh hội nhập ở thị trường tiêu dùng Việt Nam và hơn nữa tập trung vào nhóm sản phẩm tiêu dùng thương hiệu quốc gia.

\subsection{Mô hình và giả thuyết nghiên cứu}

Trong bài viết này tác giả ủng hộ quan điểm của Sweeney \& Soutar [17] vì họ đã xác định bốn yếu tố đo lường GTCN của NTD và phát triển thang đo để đo các yếu tố này cho bốn sản phẩm không bền: ngũ cốc, thanh sô cô la, và snack ngọt ở thị trường Đức dựa trên một nghiên cứu trước đó ở thị trường Úc; điều này là tương đồng với đối tượng là nhóm sản phẩm tiêu dùng mà tác giả nghiên cứu. Hơn nữa, họ còn xác định tính hiệu lực của thang đo CPV qua nhiều văn hóa khác nhau đồng thời khẳng định thang đo $\mathrm{CPV}$ có những thành tố có khả năng đo lường và tiềm năng sử dụng trên toàn thế giới. 


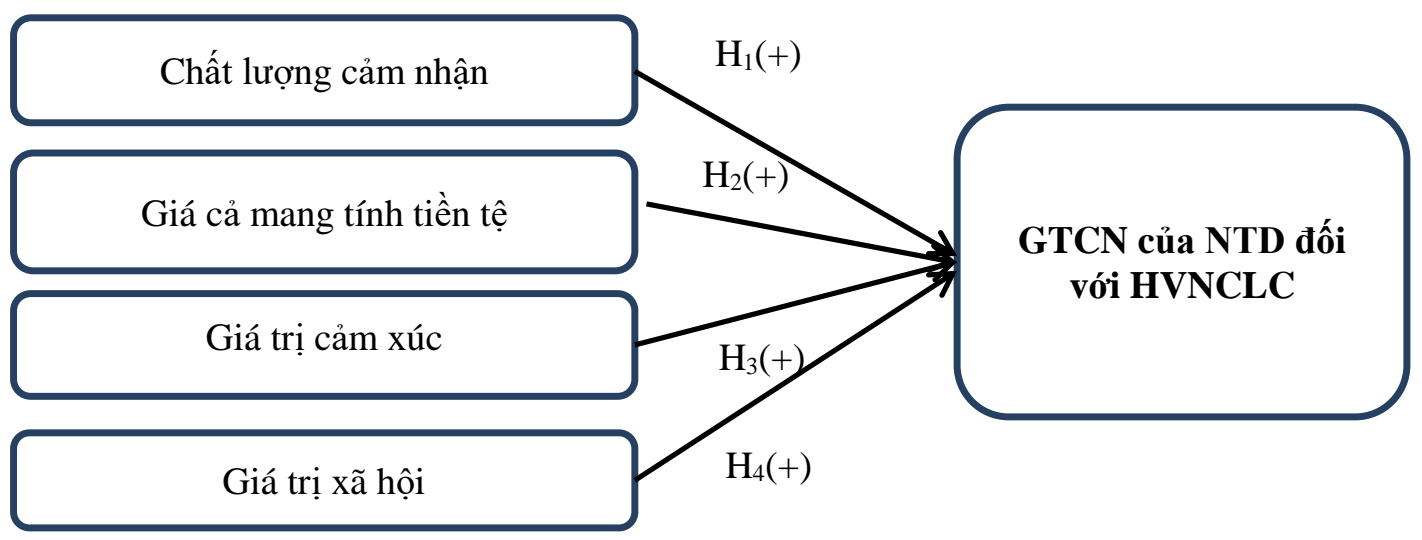

Hình 1. Mô hình nghiên cứu đề nghị

Chất lượng cảm nhận liên quan đến chức năng, lợi ích có được sản phẩm và hoạt động của sản phẩm. Trong đánh giá của NTD thì đó là sự nổi trội hay ưu thế tổng quát về chất lượng của sản phẩm. Chất lượng của sản phẩm có ảnh hưởng quan trọng đến GTCN của NTD, họ cảm nhận chất lượng của sản phẩm càng tốt thì mức GTCN tổng quát càng cao. Từ đó ta có giả thiết đầu tiên:

H1: Chất lượng cảm nhận có tác động cùng chiều lên GTCN của NTD đối với nhóm sản phẩm tiêu dùng HVNCLC.

Giá cả mang tính tiền tệ thường được hiểu là giá cả tuy nhiên trong các nghiên cứu được hiểu là giá cảm nhận hơn là giá bán của sản phẩm đó, vì NTD thường không đánh giá chính xác giá cả mà họ thường hiểu nó là rẻ, phải chăng hay đắt đỏ dựa trên giá tham khảo của riêng họ (Zeithaml [13]). Nó gắn liền với sự đánh giá liên quan đến các khía cạnh về giá cả thông qua kinh nghiệm tiêu dùng. Cụ thể là cảm nhận ở giá cả phù hợp với chất lượng, giá cả tương đối ổn định; giá cả có tính cạnh tranh; giá cả phù hợp với thu nhập của khách hàng. Từ đó ta có giả thiết thứ hai:

H2: Giá cả mang tính tiền tệ có tác động cùng chiều lên GTCN của NTD đối với nhóm sản phẩm tiêu dùng HVNCLC.

Giá trị cảm xúc liên quan tới lợi ích có được từ những cảm giác hay trạng thái xúc động do sản phẩm mang lại. Nó mô tả niềm vui thích mà sản phẩm mang lại cho khách hàng. Phản ứng cảm xúc của khách hàng đối với sản phẩm càng tốt họ càng cảm thấy vui vẻ, thoải mái hoặc hạnh phúc khi sử dụng sản phẩm thì mức GTCN tổng quát càng cao. Từ đó ta có giả thiết thứ ba:

H3: Giá trị cảm xúc có tác động cùng chiều lên GTCN của NTD đối với nhóm sản phẩm tiêu dùng HVNCLC.

Giá trị xã hội là lợi ích có được từ khả năng của sản phẩm làm tăng tính cá nhân trong xã hội. Đó là khách hàng được ghi nhận, được đề cao về địa vị, tăng vị trí trong cảm nhận của cộng đồng về bản thân, hoặc được gia nhập vào các mối quan hệ xã hội... NTD nhận thấy giá trị xã hội của bản thân được nâng cao khi tin dùng sản phẩm thì thì mức GTCN tổng quát càng cao. Từ đó ta có giả thiết thứ tư:

H4: Giá trị xã hội có tác động cùng chiều lên GTCN của NTD đối với nhóm sản phẩm tiêu dùng HVNCLC.

\section{PHƯƠNG PHÁP NGHIÊN CÚU}

Nhóm tác giả sử dụng phương pháp nghiên cứu hỗn hợp và thực hiện thông qua hai giai đoạn: (1) nghiên cứu định tính và (2) nghiên cứu định lượng.

Nghiên cứu định tính: nhóm tác giả sử dụng phương pháp nghiên cứu định tính tính nhằm thu thập phân tích và diễn giải dữ liệu không thể lượng hóa được. Cụ thể, trong trường hợp này phương pháp phỏng vấn sâu chuyên gia được áp dụng để hiệu chỉnh thang đo sau khi sử dụng phương pháp thảo luận nhóm với 21 NTD để khám phá các biến quan sát. Các chuyên gia tham gia là các giảng viên và các nhà quản lý hoạt động trong lĩnh vực: marketing, kinh doanh ngành hàng tiêu dùng, thẩm định chất lượng sản phẩm. Sau đó, nhóm tác giả tiến hành khảo sát sơ bộ với kích thước mẫu $\mathrm{n}=50$ nhằm loại các biến không phù hợp. Kết quả thang đo chính thức bao gồm 18 biến độc lập thuộc 4 nhân tố độc lập: (1) chất lượng 
cảm nhận (gồm 6 biến, ký hiệu là PQ); (2) giá cả mang tính tiền tệ (gồm 4 biến, ký hiệu là $\mathrm{MC}$ ); (3) giá trị cảm xúc (gồm 3 biến, ký hiệu EV); (4) giá trị xã hội (gồm 5 biến, ký hiệu SV); và 6 biến phụ thuộc của nhân tố GTCN tổng thể (ký hiệu CPV).

Nghiên cứu định lượng: nhóm tác giả tiến hành khảo sát, thu thập dữ liệu thông qua bảng câu hỏi được phát trực tiếp cho người tiêu dùng trên địa bàn thành phố Hồ Chí Minh. Trong trường hợp này, phương pháp lấy mẫu thuận tiện (convenience sampling) được lựa chọn với kích thước mẫu được xác định dựa vào tiêu chí sau: số lượng mẫu phù hợp cho kĩ thuật phân tích EFA theo tỉ lệ quan sát (observations)/biến đo lường (items) là $5: 1$ (Hair [3]), vậy mẫu tối thiểu cần thiết là $n 1=120(24 * 5)$; cỡ mẫu phù hợp được tính toán dựa trên tổng thể 8.224.000.000 người, với độ tin cậy $95 \%$ là $\mathrm{n} 2=\mathrm{N} / 1+$ $\mathrm{N}^{*}\left(\mathrm{e}^{2}\right)=400$. Để đáp ứng được trong giới hạn thời gian và chi phí, cũng đề phòng các tác nhân làm giảm quy mô mẫu trong quá trình khảo sát thực tể và làm sạch dữ liệu mà vẫn đảm bảo độ tin cậy cao cho đề tài tác giả chọn kích thước mẫu $\mathrm{n}=550$.

\section{KẾT QUẢ NGHIÊN CÚU VÀ THẢO LUậN}

\subsection{Tóm tắt mẫu nghiên cứu}

Tiến hành khảo sát chính thức 550 mẫu thu về 542 mẫu trong đó có 538 mẫu hợp lệ. Kết quả thống kê cho thấy: phần lớn các đáp viên có trình độ cao đẳng/đại học (chiếm 55,6\%) và tốt nghiệp THPT TCCN (chiếm 21\%). Các đối tượng tham gia phỏng vấn tập trung nhiều ở mức độ tuổi từ 18 đến 30 tuổi. Phần lớn những đáp viên này tập trung ở các nghề nghiệp như: nhân viên văn phòng $(24.9 \%)$, chủ kinh doanh $(12,3 \%)$, nội trợ $(18,4 \%)$ và sinh viên $(13,9 \%)$.

Bảng 1: Tóm tắt mẫu nghiên cứu

\begin{tabular}{|l|l|c|c|}
\hline Chỉ tiêu thống kê & & Trường hợp & Tỉ lệ phần trăm (\%) \\
\hline \multirow{5}{*}{ Trình độ } & Dưới cấp 3 & 59 & 11,0 \\
\cline { 2 - 4 } & THPT - TCCN/TCN & 113 & 21,0 \\
\cline { 2 - 4 } & ại học/cao đẳng & 299 & 55,6 \\
\cline { 2 - 4 } & Sau đại học & 67 & 12,5 \\
\hline \multirow{5}{*}{ Độ tuổi } & $(=) 23$ tuổi & 122 & 22,7 \\
\cline { 2 - 4 } & $24-30$ tuối & 197 & 36,6 \\
\cline { 2 - 4 } & $31-40$ tuồi & 95 & 17,7 \\
\cline { 2 - 4 } & $41-50$ tuồi & 82 & 15,2 \\
\cline { 2 - 4 } & Trên 50 tuổi & 42 & 7,8 \\
\hline \multirow{5}{*}{ Ngành } & Chuyên gia/chuyên viên & 18 & 3,3 \\
\cline { 2 - 4 } & Kỹ thuật viên & 27 & 5,0 \\
\cline { 2 - 4 } & iiều hành/quản lý cấp cao & 7 & 1,3 \\
\cline { 2 - 4 } & Nhân viên văn phòng & 134 & 24,9 \\
\cline { 2 - 4 } & Công nhân sản xuất & 34 & 6,3 \\
\cline { 2 - 4 } & Chủ kinh doanh riêng & 66 & 12,3 \\
\cline { 2 - 4 } & Học sinh - Sinh viên & 78 & 14,5 \\
\cline { 2 - 4 } & Nội trợ & 69 & 18,4 \\
\cline { 2 - 4 } & Khác & 60 & 11,1 \\
\hline
\end{tabular}

\subsection{Kiểm định thang đo bằng hệ số Cronbach's Alpha}

Kết quả phân tích hệ số tin cậy Cronbach's Alpha của thang đo trong lần thứ nhất cho thấy các nhân tố trong mô hình đều có hệ số Cronbach's Alpha 0,6; các hệ số tương quan biến tổng (corrected itemtotal correlation) của các thang đo này đều lớn hơn tiêu chuẩn cho phép $(>0,3)$. Tuy nhiên, biến $\mathrm{MC} 3$ của nhân tố "Giả cả mang tính tiền tệ" có hệ số Alpha khi loại biến (Alpha of Item Deleted) lớn hơn hệ số Conbach's Alpha $(0,676)$ nên nhóm tác giả loại biến ra khỏi thang đo và tiến hành chạy lại Conbach's Alpha lần 2 cho nhóm nhân tố "Giá cả mang tính tiền tệ". Như vậy, sau khi tiến hành phân tích độ tin cậy của thang đo thì tất cả 5 nhân tố với 23 biến quan sát có độ tin cậy khá tốt, có thể để đưa vào mô hình và được dùng trong phân tích EFA tiếp theo. 
Bảng 2: Tổng hợp hệ số Cronbach’s Alpha

\begin{tabular}{|l|c|c|c|}
\hline \multicolumn{1}{|c|}{ Nhân tố } & $\begin{array}{c}\text { Hệ số Cronbach's Alpha lần } \\
1\end{array}$ & $\begin{array}{c}\text { Hệ số cronbach's Alpha } \\
\text { lần 2 }\end{array}$ & Số biến \\
\hline Chất lượng cảm nhận (PQ) & 0,723 & & 6 \\
\hline Giá cả mang tính tiền tệ (MC) & 0,676 & 0,690 & 3 \\
\hline Giá trị cảm xúc (EV) & 0,759 & & 3 \\
\hline Giá trị xã hội (SV) & 0,703 & & 5 \\
\hline GTCN chung (CPV) & 0,786 & & 6 \\
\hline
\end{tabular}

\subsection{Phân tích nhân tố EFA}

Bảng 3: Kết quả EFA cuối cùng của thang đo các nhân tố độc lập

\begin{tabular}{|c|c|c|c|c|c|}
\hline \multirow{2}{*}{ Kí hiệu } & \multicolumn{4}{|c|}{ Nhóm nhân tố } & \multirow[t]{2}{*}{ Chú thích các biến } \\
\hline & 1 & 2 & 3 & 4 & \\
\hline EV2 & 0,772 & & & & Tôi có thiện cảm với các sản phẩm tiêu dùng HVNCLC. \\
\hline EV3 & 0,717 & & & & Tôi cảm giác an tâm sử dụng các sản phẩm tiêu dùng HVNCLC \\
\hline EV1 & 0,657 & & & & Tôi cảm thấy thích thú với các sản phẩm tiêu dùng HVNCLC \\
\hline SV2 & & 0,734 & & & $\begin{array}{l}\text { Tôi thấy được cải thiện hình ảnh trước bạn bè đồng nghiệp khi sử } \\
\text { dụng các sản phẩm tiêu dùng HVNCLC }\end{array}$ \\
\hline SV1 & & 0,650 & & & $\begin{array}{l}\text { Tôi cảm thấy mình là NTD thông thái khi sử dụng các sản phẩm tiêu } \\
\text { dùng HVNCLC }\end{array}$ \\
\hline SV3 & & 0,635 & & & $\begin{array}{l}\text { Các sản phẩm tiêu dùng HVNCLC tôi đã mua được mọi người đánh } \\
\text { giá cao }\end{array}$ \\
\hline SV4 & & 0,554 & & & $\begin{array}{l}\text { Mọi người ủng hộ quyết định mua các sản phẩm tiêu dùng } \\
\text { HVNCLC của tôi }\end{array}$ \\
\hline SV5 & & 0,501 & & & $\begin{array}{l}\text { Uu tiên sử dụng các sản phẩm HVNCLC là hành vi thể hiện lòng } \\
\text { yêu nước của tôi. }\end{array}$ \\
\hline PQ2 & & & 0,732 & & $\begin{array}{l}\text { Các sản phẩm tiêu dùng HVNCLC sử dụng nguồn nguyên liệu an } \\
\text { toàn }\end{array}$ \\
\hline PQ4 & & & 0,614 & & Các sản phẩm tiêu dùng HVNCLC có lợi cho sức khỏe \\
\hline $\mathrm{PQ} 3$ & & & 0,611 & & Các sản phẩm tiêu dùng HVNCLC có kiểu dáng bao bì đẹp \\
\hline PQ1 & & & 0,580 & & Các sản phẩm tiêu dùng HVNCLC có mức chất lượng ổn định \\
\hline PQ5 & & & 0,514 & & $\begin{array}{l}\text { Các sản phẩm tiêu dùng HVNCLC có chứng nhận tiêu chuẩn chất } \\
\text { lượng đáng tin }\end{array}$ \\
\hline $\mathrm{MC} 2$ & & & & 0,756 & $\begin{array}{l}\text { Các sản phẩm tiêu dùng HVNCLC có giả cả phù hợp với thu nhập } \\
\text { của tôi }\end{array}$ \\
\hline MC1 & & & & 0,741 & $\begin{array}{l}\text { Các sản phẩm tiêu dùng HVNCLC có giả cả phù hợp với chất } \\
\text { lượng }\end{array}$ \\
\hline MC4 & & & & 0,669 & Các sản phầm tiêu dùng HVNCLC có giả cả ồn định \\
\hline \multicolumn{5}{|l|}{ KMO } & $\mathbf{0 , 8 9 9}$ \\
\hline \multicolumn{5}{|c|}{ Chỉ số dừng nhân tố } & $\mathbf{1 , 0 1 4}$ \\
\hline \multicolumn{5}{|c|}{ Tổng phương sai trích } & $\mathbf{5 4 , 7 4 9}$ \\
\hline
\end{tabular}

Các biến đã đạt yêu cầu trong phân tích Cronbach's Alpha đều được sử dụng trong phân tích EFA. Chỉ số KMO (Kaiser-Meyer-Olkin Measure of Sampling Adequacy) được dùng để phân tích sự thích hợp của các nhân tố. Phân tích chỉ được sử dụng khi có chỉ số KMO lớn hơn 0,55.

Tiến hành chạy 2 lần phân tích nhân tố cho các biến độc lập. Kết quả như sau:

Kết quả EFA lần thứ nhất cho thấy có 4 nhân tố được rút trích tại Eigenvalue là 1,025 và phương sai trích được $52,914 \%$ với chỉ số KMO là 0,902 . Như vậy việc phân tích nhân tố là phù hợp. Tuy nhiên, ta thấy biến quan sát PQ6 có hệ số tải nhân tố (factor loading) không thỏa điều kiện nên ta loại biến này. 
Kết quả EFA lần thứ hai, sau khi loại biến PQ6 thì EFA rút trích được 4 nhân tố với chỉ số Eigenvalue là 1,014 và phương sai trích được $54,749 \%$ với chỉ số KMO là 0,899 . Như vậy việc phân tích nhân tố là phù hợp. Đồng thời, tất cả các biến quan sát đều có hệ số tải nhân tố đạt yêu cầu, do đó phân tích EFA hoàn tất và ghi nhận kết quả phân tích lần thứ 2 là kết quả cuối cùng

Kết quả chung được thể hiện qua bảng 3 kết quả EFA cuối cùng của thang đo các nhân tố độc lập.

Và trong 2 lần chạy EFA, các giá trị Sig. đều $<0,05$ điều đó chứng tỏ các biến có tương quan với nhau trong tổng thể.

Tương tự như phân tích các biến độc lập, tiến hành phân tích nhân tố cho biến phụ thuộc. Kết quả được thể hiện ở bảng 4 .

Bảng 4: Chỉ số KMO và Bartlett's Test (biến phụ thuộc)

\begin{tabular}{|l|l|l|}
\hline \multicolumn{2}{|l|}{ Kaiser-Meyer-Olkin Measure of Sampling Adequacy. } & 0,809 \\
\hline \multirow{3}{*}{ Bartlett's Test of Sphericity } & Approx. Chi-Square & 838,405 \\
\cline { 2 - 3 } & Df & 15 \\
\cline { 2 - 3 } & Sig. & .000 \\
\hline
\end{tabular}

Chỉ số $\mathrm{KMO}=0,809>0,6$ và significance $\mathrm{P}$. Value $=0,000<0,05$ cho thấy đánh giá chung GTCN của NTD phù hợp để phân tích nhân tố và các biến quan sát trong biến phụ thuộc có tương quan với nhau.

\subsection{Phân tích nhân tố khẳng định $\mathrm{CFA}$}

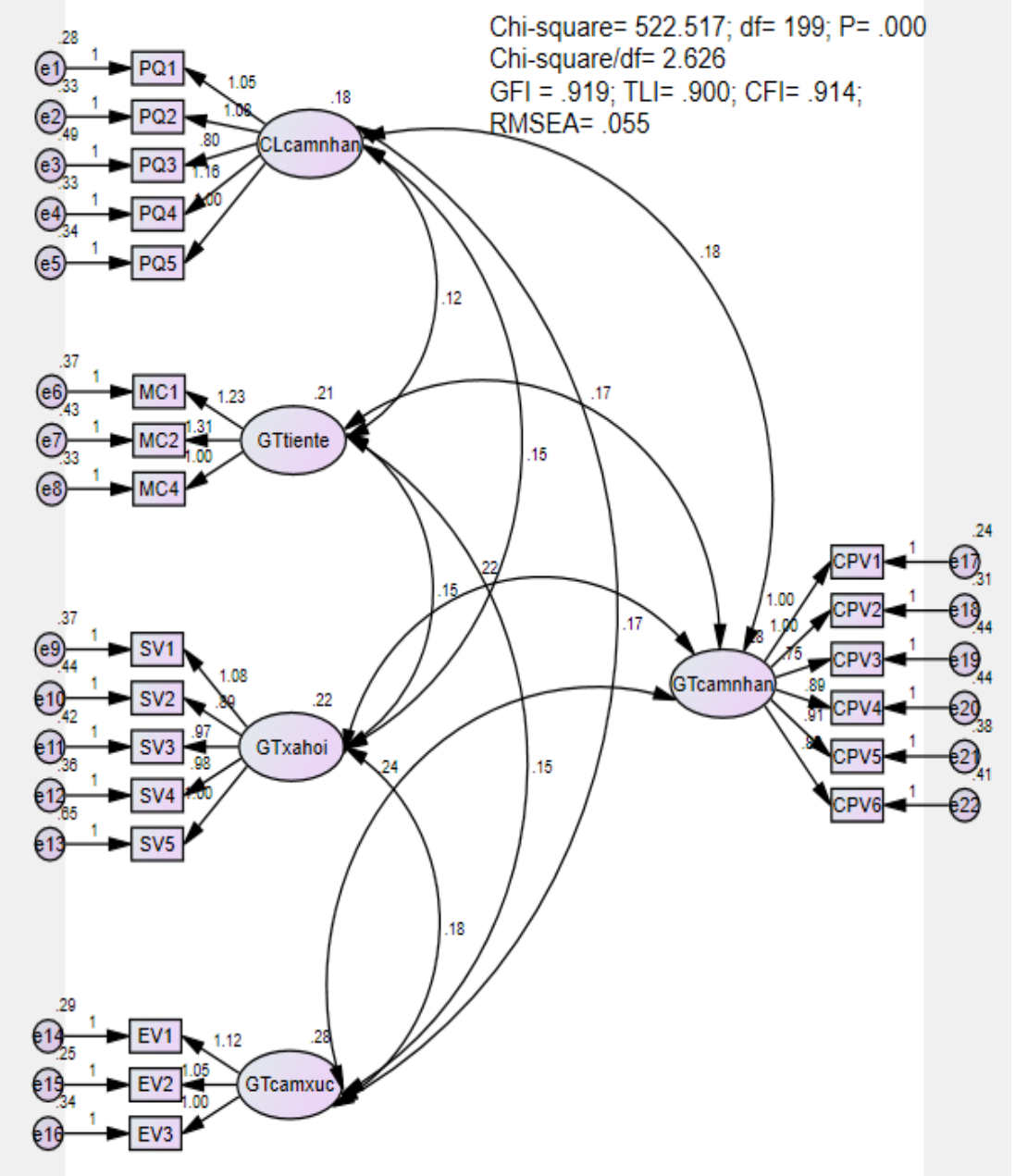

Hình 2: Mô hình CFA thang đo các khái niệm 
Tiến hành phân tích nhân tố khẳng định các biến thuộc các biến độc lập và phụ thuộc. TLI $=0,900$; $\mathrm{CFI}=0,914$ đều lớn hơn 0,9 . Giá trị RMSEA $=0,055<0,08$ do đó mô hình đạt độ thích hợp tốt với dữ liệu khảo sát.

\subsection{Kiểm định mối liên hệ nhân quả trong mô hình cấu trúc tuyến tính}

Bảng 5: Kết quả kiểm định mối quan hệ nhân quả (chưa chuẩn hóa)

\begin{tabular}{|lll|rrrr|}
\hline & & & Estimate & S.E. & \multicolumn{1}{l|}{ C.R. } & \multicolumn{1}{c|}{ P } \\
\hline Gtcamnhan & $<---$ & CLcamnhan & 0,200 & 0,112 & 1,786 & 0,074 \\
Gtcamnhan & $<---$ & Gctiente & 0,061 & 0,077 & 0,794 & 0,427 \\
Gtcamnhan & $<---$ & Gtxahoi & 0,488 & 0,118 & 4,139 & $* * *$ \\
Gtcamnhan & $<---$ & GTcamxuc & 0,391 & 0,082 & 4,776 & $* * *$ \\
\hline
\end{tabular}

Dựa vào kết quả kiểm định mối quan hệ nhân quả (chưa chuẩn hóa và đã chuẩn hóa) có thể kết luận Giá trị cảm xúc, Giá trị xã hội thực sự có ảnh hưởng thuận chiều đến giá trị cảm nhận một cách có ý nghĩa với mức độ tin cậy $95 \%$. Riêng yếu tố chất lượng cảm nhận có mối quan hệ tương quan thuận chiều với giá trị cảm nhận một cách có ý nghĩa với mức độ tin cậy $90 \%$. Đồng thời chưa đủ bằng chứng cho thấy yếu tố giá cả mang tính chất tiền tệ trong trường hợp này thực sự có tác động đến giá trị cảm nhận của người tiêu dùng đối với HVNCLC.

Bảng 6: Kết quả kiểm định Bootstrap

\begin{tabular}{|lll|crrrrr|}
\hline Parameter & & & Estimate & SE & SE-SE & Mean & Bias & SE-Bias \\
\hline GTcamnhan & $<---$ & CLcamnhan & $\mathbf{0 , 1 5 9}$ & 0,156 & 0,008 & $\mathbf{0 , 1 4 9}$ & $\mathbf{- 0 , 0 1 0}$ & $\mathbf{0 , 0 1 1}$ \\
GTcamnhan & $<---$ & GTtiente & $\mathbf{0 , 0 5 2}$ & 0,070 & 0,003 & $\mathbf{0 , 0 5 7}$ & $\mathbf{0 , 0 0 5}$ & $\mathbf{0 . 0 0 5}$ \\
GTcamnhan & $<---$ & GTxahoi & $\mathbf{0 , 4 2 6}$ & 0,134 & 0,007 & $\mathbf{0 , 4 2 8}$ & $\mathbf{0 , 0 0 2}$ & $\mathbf{0 , 0 0 9}$ \\
GTcamnhan & $<---$ & GTcamxuc & $\mathbf{0 , 3 8 9}$ & 0,102 & 0,005 & $\mathbf{0 , 3 9 3}$ & $\mathbf{0 , 0 0 4}$ & $\mathbf{0 , 0 0 7}$ \\
\hline
\end{tabular}

Đồng thời, kết quả kiểm định Bootstrap cũng cho thấy độ chệch giữa trung bình các ước lượng Bootstrap (Mean) với ước lượng bình thường bằng phương pháp ML (Estimate) là không đáng kể (khá nhỏ). Các ước lượng (Estimate) trong mô hình có thể tin cậy được. Theo đó, giá trị xã hội sẽ có tác động mạnh nhất đến giá trị cảm nhận, kế đến là giá trị cảm xúc và cuối cùng là chất lượng cảm nhận.

Bảng 7: Tổng hợp kết quả kiểm định giả thuyết

\begin{tabular}{|c|c|c|c|c|}
\hline $\mathbf{H}$ & Phát biểu & $\begin{array}{l}\text { Hệ số hồi quy } \\
\text { (Chuẩn hóa) }\end{array}$ & $\mathbf{P}$ & Kết quả \\
\hline H1 & $\begin{array}{l}\text { Chất lượng cảm nhận có tác động cùng chiều lên giá trị } \\
\text { cảm nhận của người tiêu dùng đối với nhóm sản phâmm } \\
\text { tiêu dùng HVNCLC }\end{array}$ & 0,159 & 0,074 & Chấp nhận \\
\hline H2 & $\begin{array}{l}\text { Giá cả mang tính tiền tệ có tác động cùng chiều lên giá } \\
\text { trị cảm nhận của người tiêu dùng đối với nhóm sản } \\
\text { phẩm tiêu dùng HVNCLC }\end{array}$ & 0,052 & 0,427 & $\begin{array}{l}\text { Chưa đủ bằng } \\
\text { chứng để kết } \\
\text { luận }\end{array}$ \\
\hline H3 & $\begin{array}{l}\text { Giá trị cảm xúc có tác động cùng chiều lên giá trị cảm } \\
\text { nhận của người tiêu dùng đối với nhóm sản phâm tiêu } \\
\text { dùng HVNCLC }\end{array}$ & 0,389 & 0,000 & Chấp nhận \\
\hline H4 & $\begin{array}{l}\text { Giá trị xã hội có tác động cùng chiều lên giá trị cảm } \\
\text { nhận của người tiêu dùng đối với nhóm sản phâm tiêu } \\
\text { dùng HVNCLC }\end{array}$ & 0,426 & 0,000 & Chấp nhận \\
\hline
\end{tabular}




\section{KẾT LUẬN VÀ MỘT SỐ HÀM Ý QUẢN TRI}

\subsection{Một số hàm ý quản trị nhằm nâng cao giá trị cảm nhận của người tiêu dùng}

Giá trị cảm nhận của NTD đối với nhóm sản phẩm tiêu dùng chỉ ở mức trung bình khá $(3,703)$ do đó cần nhiều nỗ lực để nâng cao mức giá trị cảm nhận đối với nhóm sản phẩm này.

Kết quả kiểm định SEM cho thấy có ba nhóm nhân tố cấu thành GTCN của NTD. Trong đó nhân tố ảnh hưởng mạnh nhất là giá trị xã hội đạt giá trị Estimate $=0,426$, tiếp đó là giá trị cảm xúc đạt giá trị Estimate $=0,389$, cuối cùng là nhân tố chất lượng cảm nhận đạt giá trị Estimate $=0,159$. Đây là cơ sở ưu tiên thực hiện các giải pháp cho đề tài để các doanh nghiệp xây dựng được chiến lược tối đa hóa GTCN cho NTD. Đồng thời, các trị số điểm đánh giá của NTD và thực trạng từ dữ liệu thứ cấp được tác giả phân tích làm cơ sở đề xuất các giải pháp được trình bày bên dưới.

\section{Về giá trị xã hội}

Trong thực trạng cạnh tranh gay gắt như hiện nay và với nguồn lực có giới hạn thì việc phát huy và nâng mức giá trị cảm nhận cho hàng hóa về mặt xã hội cho người tiêu dùng là cách làm hiệu quả và tiết kiệm chi phí cho các doanh nghiệp Việt Nam. Ngoài việc tuyên truyền vận động sử dụng HVNCLC bằng tình cảm yêu nước, doanh nghiệp cần nhận thức rõ vai trò của truyền thông tác động từng ngày vào suy nghĩ của NTD; áp dụng những thông lệ kinh doanh trên cơ sở tôn trọng con người, tăng cường trách nhiệm với cộng đồng và môi trường.

\section{Về giá trị cảm cảm xúc}

Các doanh nghiệp phải tập trung vào việc gia tăng các yếu tố về cảm xúc như là sự thích thú trong mua sắm, tạo nên mối thiện cảm và sự an tâm khi sử dụng các sản phẩm tiêu dùng; nắm bắt được những thay đổi về tâm lý, sở thích và thu nhập của NTD nội địa để làm nền tảng xây dựng các chiến lược về tiếp thị, truyền thông; xây dựng kiểu dáng, bao bì cho phù hợp với những thay đồi về thị hiếu của NTD; cải thiện về chất cũng như về lượng các chương trình bán hàng và trưng bày hàng hóa.

\section{Về chất lượng cảm nhận}

Sáng tạo và đổi mới, không rập khuôn theo những mẫu mã sản phẩm truyền thống có từ trước; chú trọng vào việc gìn giữ cũng như bảo vệ thiết kế của doanh nghiệp để tránh việc làm nhái làm giả hàng kém chất lượng; doanh nghiệp khi thiết kế bao bì cũng phải thật chú ý đến những xu hướng tiện dụng, an toàn và thân thiện với môi trường.

Củng cố thị trường nguyên vật liệu đầu vào cho sản xuất bằng cách ký các hợp đồng với nhiều bạn hàng để đảm bảo cung ứng, đủ loại cho sản xuất, đồng thời phải tạo ra nhưng bạn hàng lâu dài, uy tín để đảm bảo chất lượng nguyên vật liệu là điều mà các doanh nghiệp cần phải hướng đến.

Với các sản phẩm tiêu dùng sử dụng nguyên vật liệu trong nước như: thịt và các sản phẩm từ thịt; sữa và các sản phẩm từ sữa; thức ăn, đồ uống chế biến để ăn ngay; thực phẩm đông lạnh... doanh nghiệp cần chủ động trong việc xác định nguồn cung lâu dài đảm bảo chất lượng, tự xây dựng chuỗi cung cấp hoặc đầu tư nuôi - trồng - sản xuất để có nguồn nguyên liệu sản xuất an toàn tạo lòng tin cho NTD.

Có hình thức xử phạt công khai, nghiêm khắc với những trường hợp sử dụng giấy chứng nhận giả làm thiệt hại cho NTD.

Ngoài ra, khi xảy ra tình trạng các sản phẩm bị NTD phản ảnh cần phải phải có thái độ xác minh, nhận lỗi nếu có, mạnh dạn đưa ra thông tin những sản phẩm bị lỗi, nguyên nhân bị lỗi, biểu hiện của lỗi và quan trọng là chấp nhận thu hồi những sản phẩm này.

\subsection{Kết luận}

Dựa trên cơ sở lý thuyết về giá trị cảm nhận của khách hàng và các nghiên cứu trước đây có liên quan được công bố, đề tài này đã xác định được 3 thành phần cấu thành giá trị cảm nhận của người tiêu dùng đối với nhóm sản phẩm tiêu dùng HVNCLC: (1) Giá trị xã hội, (2) Giá trị cảm xúc, (3) Chất lượng cảm nhận. Các nhân tố này đều tác động cùng chiều lên giá trị cảm nhận của người tiêu dùng. Kết quả này cũng ủng hộ kết quả của các nghiên cứu gần đây về việc tìm ra các nhân tố quyết định đến việc ung hộ các sản phẩm tiêu dùng HVNCLC của người tiêu dùng như: Báo cáo công bố ngày 23-04-2016 Nielsen Global Brand Origin Survey, Q3-2015; "Các yếu tố tác động đến việc NTD chọn mua hàng thực phẩm Việt Nam" - Ngô Thái Hưng, Tạp chí Khoa học - Số 01 (2013): 48 - 56. Bên cạnh đó, đề tài đã đóng góp bộ thang đo có giá trị có thể ứng dụng cho việc đo lường giá trị cảm nhận của người tiêu dùng 
đối với các mặt hàng tiêu dùng ở thị trường Việt Nam nhờ sự điều chỉnh của các chuyên gia và đạt kết quả kiểm định định lượng cao. Điều này còn cho thấy mô hình nghiên cứu GTCN có thể là một công cụ hữu ích để xây dựng chiến lược cạnh tranh cho các doanh nghiệp khi được đo lường tốt.

Do hạn chế về thời gian và tài chính trong việc nghiên cứu, nghiên cứu này chỉ được thực hiện trên 538 người tiêu dùng trong khu vực thành phố Hồ Chí Minh để đưa ra giải pháp. Các đối tượng được khảo sát chưa thật sự đồng đều về địa điểm, giới tính, trình độ học vấn, nghề nghiệp nên tính đại diện cho tổng thể chưa cao.

Đề tài có thể mở rộng phạm vi nghiên cứu áp dụng cho các nhóm sản phẩm HVNCLC khác để có chính sách phát triển toàn diện thương hiệu quốc gia. Đây cũng là hướng cho các nghiên cứu tiếp theo.

\section{TÀI LIỆU THAM KHẢO}

[1] Bolton, Ruth N. and James H. Drew (1991), "A Multistage Model of Customers' Assessments of Service Quality and Value", Journal of Consumer Research, 17(4), 375-384.

[2] Grewal, D., Monroe, K.B., \& Krishnan, R. (1998), "The effects of price-comparison advertising on buyers' perceptions of acquisition value, transaction value and behavioral intentions", Journal of Marketing, 62 (Apr), 46-59.

[3] Hair, Jr. J.F, Anderson, R.E., Tatham, R.L. \& Black, W.C. (1998), Multivariate Data Analysis, Prentical-Hall International Inc.

[4] Hoàng Trọng, Chu Nguyễn Mộng Ngọc (2005), Phân tích dũ liệu nghiên cứu với SPSS, NXB Thống Kê, Hà Nội.

[5] Lapierre, J. (2000), "Customer-perceived value in industrial contexts", The Journal of Business \& Industrial Marketing. 15(2/3), 122-145.

[6] Lê Văn Hoàng \& Nguyễn Thị Ngọc Huỳnh (2014) "Đo luòng GTCN của khách hàng cá nhân đối với sản phẩm xe máy điện tại vùng đông nam bộ", Tạp chí Khoa học Lạc Hồng - Tập 1, Số 1 (2014): 12-14.

[7] Monroe, Kent B. (1990), Pricing: Making Profitable Decisions, McGraw-Hill, New York.

[8] Ngô Thái Hưng, (2013), Các yếu tố tác động đến việc NTD chọn mua hàng thục phẩm việt nam, Tạp chí Khoa học - Số 01 (2013): 48 - 56

[9] Nguyễn Đình Thọ, Nguyễn Thị Mai Trang (2007), Nghiên cứu khoa học Marketing - Úng dụng mô hình cấu trúc tuyến tính SEM, NXB Đại học quốc gia Tp. Hồ Chí Minh, Tp.HCM.

[10] Nguyễn Đình Thọ, Nguyễn Thị Mai Trang (2007), Nghiên cứu thị truờng, NXB Đại học quốc gia Tp. Hồ Chí Minh, Tp.HCM.

[11] Nguyễn Ngọc Châu (2010), "Giá trị dành cho khách hàng được cảm nhận và chiến luợc cạnh tranh của Công ty TNHH Gas Petrolimex Sài Gòn, Trường Đại học Kinh tế TP. HCM, Luận văn thạc sỹ.

[12] Nguyễn Thị Hồng Thắm (2009), Nghiên cứu GTCN của khách hàng đối với chuỗi siêu thị Co.op Mart, Trường Đại học Kinh tế TP. HCM, Luận văn thạc sỹ.

[13] Parasuraman A., Valarie A. Zeithaml, and Leonard L. Berry (1985), "A Conceptual Model of Service Quality and Its Implications for Future Research", Journal of Marketing, 49(4), 41-50.

[14] Parasuraman, A., \& Grewal, D. (2000), "The impact of technology on the quality-value-loyalty chain: a research agenda", Journal of the Academy of Marketing Science, 28(1), 168-74.

[15] Philip Kotler \& Kevin Lane Keller (2006), Marketing management, $12^{\text {th }}$ Edition, Pearson Prentice Hall, New Jersey. 
[16] Sheth, J.N., Newman, B.I. and Gross, B.L. (1991a), “Why we buy what we buy: a theory of consumption values”, Journal of Business Research, Vol. 22, pp. 159-70.

[17] Sweeney, Jillian C. and Geoffrey N. Soutar (2001), “Consumer-Perceived Value: The Development of a Multiple Item Scale,” Journal of Retailing, 77(2), 203-220.

[18] Woodall, Tony (2003), "Conceptualising 'Value for the Customer': A Structural, Attributional and Dispositional Perspective", Academy of Marketing Science Review, 2003(12), 1-42.

[19] Woodruff, Robert B. (1997), "Customer Value: The Next Source for Competitive Edge", Journal of the Academy of Marketing Science, 25(2), 139-153.

Ngày nhận bài: 07/01/2017

Ngày chấp nhận đăng: 07/05/2017 